\title{
CD24 Contributes to Treatment Effect in ABC-DLBCL Patients with R-CHOP Resistance
}

\author{
Li-Yan Qiao',* \\ Han-Bing $\mathrm{Li}^{2, *}$ \\ Yue Zhang $\mathbb{D}^{2}$ \\ Di Shen $\mathbb{D D}^{2}$ \\ Peng $\mathrm{Liu}^{3}$ \\ Yi-Qun Che $\mathbb{D}^{2}$
}

'Department of Stomatology, Beijing Tongren Hospital, Capital Medical University, Beijing, 100730, People's Republic of China; ${ }^{2}$ Department of Clinical Laboratory, National Cancer Center/National Clinical Research Center for Cancer/Cancer Hospital, Chinese Academy of Medical Sciences and Peking Union Medical College, Beijing, I0002I, People's Republic of China; ${ }^{3}$ Department of Medical Oncology, National Cancer Center/ National Clinical Research Center for Cancer/Cancer Hospital, Chinese Academy of Medical Sciences and Peking Union Medical College, Beijing, I0002I, People's Republic of China

*These authors contributed equally to this work

Correspondence: Yi-Qun Che Department of Clinical Laboratory, National Cancer Center/National Clinical Research Center for Cancer/Cancer Hospital, Chinese Academy of Medical Sciences and Peking Union Medical College, Beijing, I0002I, People's

Republic of China

$\mathrm{Tel} / \mathrm{Fax}+86-10-87788746$

Email cyq@cicams.ac.cn

Peng Liu

Department of Medical Oncology, National Cancer Center/National Clinical Research Center for Cancer/Cancer Hospital, Chinese Academy of Medical Sciences and Peking Union Medical College, Beijing, I0002I, People's

Republic of China

$\mathrm{Tel} / \mathrm{Fax}+86-10-87788507$

Email13910216310@163.com
Purpose: Diffuse large B-cell lymphoma (DLBCL) is the most common non-Hodgkin's lymphoma and of which the prognosis of activated B-cell-like (ABC) subtype is poor. Although R-CHOP significantly improves the survival of patients with DLBCL, $20 \%$ to $40 \%$ of patients were resistant to R-CHOP therapy. Thus, screening for candidate therapeutic targets for R-CHOP resistant patients is urgent. The previous researches have shown that $\mathrm{CD} 24$ is related to the development, invasion, and metastasis of cancer. Our project aims to clarify the relationship between CD24 and ABC-DLBCL.

Patients and Methods: The expression of CD24 mRNA in 118 ABC-DLBCL cases treated with R-CHOP was detected by RNAscope, and the relationship between CD24 expression and R-CHOP treatment response was analyzed. The correlation between CD24 expression and treatment efficiency was further analyzed by data downloaded from the Gene Expression Omnibus (GEO) database. The association between CD24 expression and immune response was conducted using Cell-type Identification By Estimating Relative Subsets Of RNA Transcripts (CIBERSORT) methodology and Gene Ontology (GO) biological process (BP) analysis.

Results: The positive expression rate of CD24 mRNA in ABC-DLBCL patients was 38.1\% (45/118). Complete Response (CR) rate was significantly higher in patients with CD24 high expression than those with $\mathrm{CD} 24$ low expression $(P=0.039 ; 44.4 \%$ vs $26.0 \%)$. CR rate was significantly different between CD24 high and low expression groups in the analysis of GEO datasets $(P=0.003 ; 83.2 \%$ vs $58.0 \%)$. The CD24 high expression patients had significantly lower proportions of $\mathrm{T}$ cells and nonspecific immune cells in the CIBERSORT analysis. In addition, T-helper 2 cell differentiation and monocyte chemotaxis were repressed in CD24 high expression group in the GO BP analysis.

Conclusion: $\mathrm{CD} 24$ was correlated with better R-CHOP treatment response and tumor immunosuppression in ABC-DLBCL. CD24 may be a promising signal in treatment and prognosis evaluation in ABC-DLBCL patients.

Keywords: ABC-DLBCL, CD24, RNA in situ hybridization, treatment response, tumor immunosuppression

\section{Introduction}

Diffuse large B-cell lymphoma (DLBCL), an aggressive lymphoma, is the most common form of non-Hodgkin lymphomas (NHL), accounting for approximately $35 \%$ of all new NHLs. ${ }^{1}$ DLBCL, as a heterogeneous disease, can be divided into germinal center B-cell like (GCB) and activated B-cell like (ABC) subtypes according to cell-of-origin, with about $10-15 \%$ of cases being unclassifiable. ${ }^{2}$ The ABC DLBCL relied on regulation of BCR-dependent activation of $N F-\kappa B$ and had poorer prognosis following immunochemotherapy. ${ }^{3}$ Currently, R-CHOP 
(rituximab plus cyclophosphamide, doxorubicin, vincristine, and prednisone) therapy is the standard treatment for DLBCL. Although first-line treatment of DLBCL is curative for some patients, $20 \%$ to $40 \%$ of patients still fail to achieve remission or experience relapse. ${ }^{4}$ For patients who are unable to receive high-dose chemotherapy and hematopoietic stem-cell transplantation as second-line therapy, the prognosis is inferior. ${ }^{5}$ Hence, the identification of effective ABC-DLBCL next line therapeutic targets and biomarkers for therapeutic evaluation is of paramount importance.

Previous research has found that CD24 is overexpressed in a variety of cancers and associated with the development, invasion, and metastasis of cancer cells. ${ }^{6}$ CD24, located on chromosome 6q21, encodes a glycosylphosphatidylinositol-linked cell surface glycoprotein. ${ }^{7}$ CD24 has been proved to be correlated with drug resistance and poor prognosis, and may be a potential therapy target in a variety of cancers. ${ }^{8-10}$ However, there are few papers on the role of CD24 in ABC-DLBCL. Therefore, we identified CD24 as a candidate molecule to conduct follow-up studies.

In this study, we initially analyzed the positive expression rate of CD24 in ABC-DLBCL. Then we investigated the association between $\mathrm{CD} 24$ expression and clinicopathological parameters in patients with ABC-DLBCL, and further analyzed $\mathrm{CD} 24$ expression and treatment response using GEO database. Subsequently, the functional mechanism of CD24 in ABC-DLBCL was further analyzed by utilizing certain bioinformatics methods.

\section{Materials and Methods}

\section{Patients}

Collect the tumor tissues of 118 newly diagnosed ABCDLBCL patients treated by the National Cancer Center/ Cancer Hospital, Chinese Academy of Medical Sciences (Beijing, China). The patients were enrolled according to the following criteria: (1) ABC-DLBCL diagnosed by pathology; (2) 6-8 cycles of R-CHOP treatment; (3) complete clinical prognosis data; (4) no heart, liver, renal, digestive and metabolic disorders. All patients provided written informed consent. This study was approved by Cancer Hospital, Chinese Academy of Medical Sciences ethics committee, and that it was conducted in accordance with the Declaration of Helsinki. Clinical information for each patient was obtained from medical records.

\section{Preparation of Tissue Microarray}

The collected tissues were removed from $-80^{\circ} \mathrm{C}$ and fixed in formalin, then embedded by paraffin. The tissue block was used to be cut into $4 \mu \mathrm{m}$ thick sections and then HE stained, and the tumor area was marked under the microscope. Tumor areas of all tissues were prepared as wax blocks and re-embedded. Two tissue points were taken from each tumor tissue, and the prepared wax blocks were cut into $5 \mu \mathrm{m} / \mathrm{sheet}$ tissue microarray for later use.

\section{Detection of CD24 mRNA by RNA in situ Hybridization}

RNA in situ hybridization was used to detect the prepared tissue chips. The human gene CD24 probe (ACD \# 313021) was purchased from Advanced Cell Diagnostics (ACD, ACD, Newark, LA). The tissue chip was dewaxed with xylene and hydrated with gradient ethanol; then, endogenous peroxidase was blocked with hydrogen peroxide for 10 minutes, and was repaired in the target repair solution for 15 minutes. The sections were then subjected to RNAscope probe hybridization and amplification, and DAB was used for color development, hematoxylin counterstaining, gradient ethanol dehydration, xylene transparent, and mounting. The whole processes were performed according to the instructions of the RNAscope $2.5 \mathrm{HD}$ detection kit (BROWN) (ACD \#322300). High-precision scanning of the tissue microarray was conducted by a NanoZoomer (Hamamatsu Corporation, Japan) pathology scanner, and blind evaluation was performed by two pathologists. The mRNA signal score was determined semi-quantitatively and the signal intensity and the proportion of positive cells were jointly determined. Signal intensity was divided into 0 (negative), 1 (weak positive), 2 (medium positive), 3 (strong positive); the proportion of positive cells was $0(0 \%), 1(1 \% \sim 20 \%), 2(20 \% \sim 50 \%)$, $3(51 \% \sim 100 \%)$. The product of the tumor cell signal intensity score and the positive cell ratio score was the final score of the specimen. A final score of $>3$ was considered positive for CD24 mRNA expression.

\section{Microarray Data Preprocessing}

Gene expression data and clinical data from the GSE31312 were retrieved from the Gene Expression Omnibus (GEO) database (platform GPL570). Raw microarray data of gene chips were normalized by using the MAS5.0 algorithm provided by "affy" package of R software. Furthermore, the "sva" package of R software was used to remove the 
batch effect and we obtained a dataset containing 199 ABC-DLBCL samples. The lower $25 \%$ of the relative expression of $\mathrm{CD} 24$ was regarded as the low expression group and the rest as the high expression group for subsequent analysis.

\section{Data Statistics and Bioinformatics Analysis}

Statistical analysis was performed using SPSS 22.0 software. The $\chi^{2}$ test was used to compare the relationship between gene expression and clinical parameters. Celltype Identification By Estimating Relative Subsets Of RNA Transcripts (CIBERSORT) and the LM22 signature matrix were used to assess the lymphocyte distribution. Gene Ontology (GO) biological process (BP) gene sets were analyzed to explain the functional mechanism. All statistical tests were two-sided, and considered significant when $\mathrm{p}$ values were $<0.05$.

\section{Results}

\section{Patients in ABC-DLBCL with CD24 High Expression Had Better R-CHOP Treatment Response}

We performed RNA in situ hybridization on paraffin tissue of 118 newly diagnosed ABC-DLBCL patients who received $\mathrm{R}-\mathrm{CHOP}$ treatment. The results showed that the positive rate of CD24 mRNA expression was $38.1 \%$ (45/ 118) (Figure 1). Newly diagnosed ABC-DLBCL patients underwent standard R-CHOP first-line treatment, and follow-up evaluations were performed regularly. The high expression of CD24 in ABC-DLBCL was associated with better treatment response $(P=0.039)$ (Table 1). The CD24 positive expression group had significant higher Complete Response (CR) rate than that with CD24 negative expression (44.4\% vs $26.0 \%$ ).

\section{CD24 High Expression Was Associated with Better Treatment Response in $A B C-D L B C L$ Patients from the GEO Database}

A total of 199 patients with ABC-DLBCL were enrolled from GSE31312 dataset in the GEO database to further analyze the $\mathrm{CD} 24$ expression and treatment response. The lower $25 \%$ of relative expression of CD24 was regarded as the low expression group and the rest as the high expression group for analysis. It was identified that the CD24 expression level was significantly different in treatment response $(P=0.003)$. The $\mathrm{CR}$ rate was higher in the CD24 high expression patients than in the low expression patients $(83.2 \%$ vs $58.0 \%)$. Nevertheless, gender, age, stage, ECOG score, extranodal involvement, LDH level had no significant difference between the two groups (Table 2). The results from the GEO database were in accordance with our analysis of the cases.

\section{CD24 High Expression Repressed Relative Abundance of Tumor-Infiltrating Immune Cell Populations Determined by the CIBERSORT Methodology}

The CIBERSORT and LM22 signature matrix were used together to estimate proportions of twenty-two immune cell types in samples from each training cohort patient, and to evaluate differences in the proportions of each immune cell type between the high and low expression groups. Compared with the low expression patients, the CD24 high expression patients had higher proportions of $\mathrm{B}$ cells naive, $\mathrm{B}$ cells memory and $\mathrm{T}$ cells CD4 memory resting. Interestingly, the proportions of $\mathrm{T}$ cells $\mathrm{CD} 8$, macrophages M2, dendritic cells activated were significantly decreased in the CD24 high expression group (Figure 2), which enlightened us in tumor microenvironment and immune tolerance.

\section{CD24 High Expression Inhibited Immune Cell Response in GO BP Analysis}

We analyzed the changes in biological process in different groups through Gene Set Variation Analysis (GSVA). We found 19 pathways with significant differences $(P<$ 0.001 ) between the CD24 low and high expression group. The T-helper 2 cell differentiation, positive regulation of transmembrane transport, positive regulation of cation transmembrane transport, membrane repolarization during action potential, and positive regulation of monocyte chemotaxis were the top 5 terms with the most significant differences, and were all attenuated significantly in the high expression group (Figure 3). T-helper 2 plays an important role in tumor immunity, and monocytes, the precursors of macrophages and dendritic cells, are also important elements in recognizing and killing tumor cells. Thus, we speculated that the tumor immune response was suppressed in the CD24 high expression group. 


\section{positive}
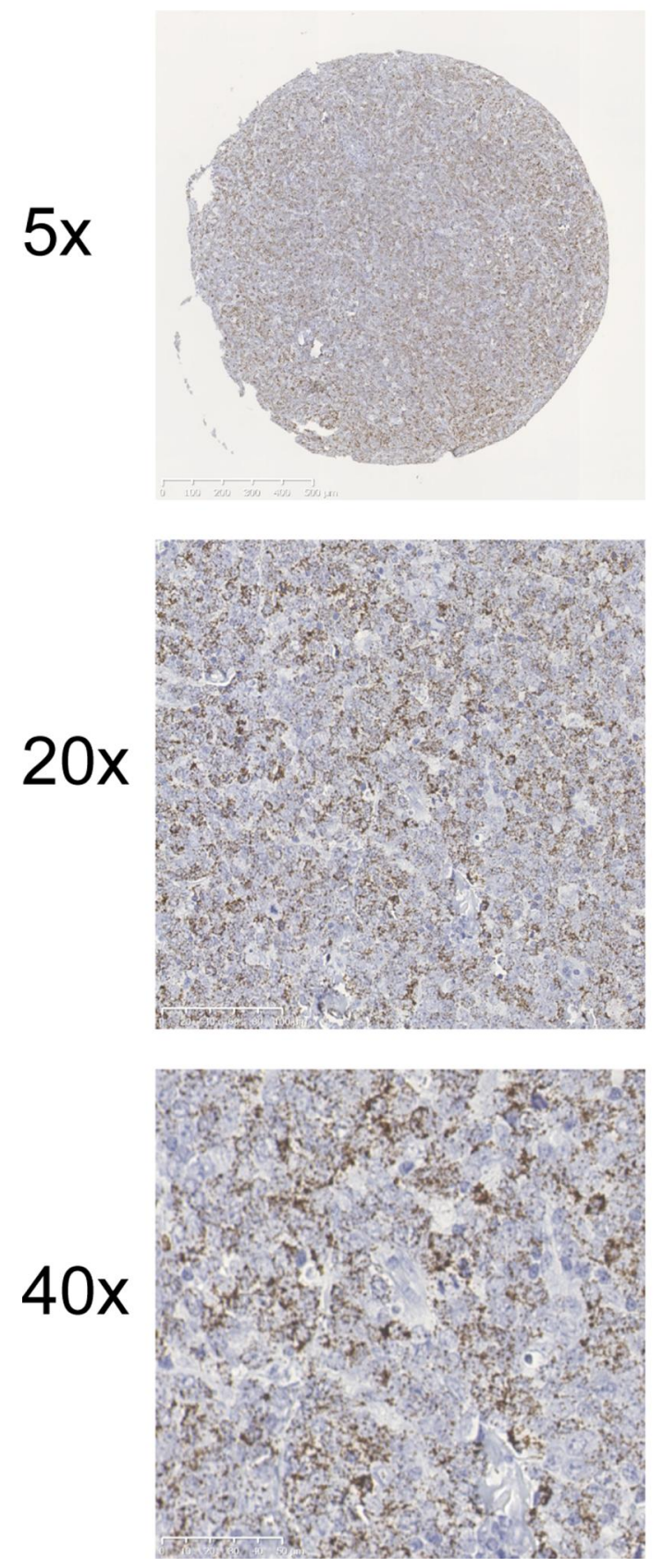

\section{negative}
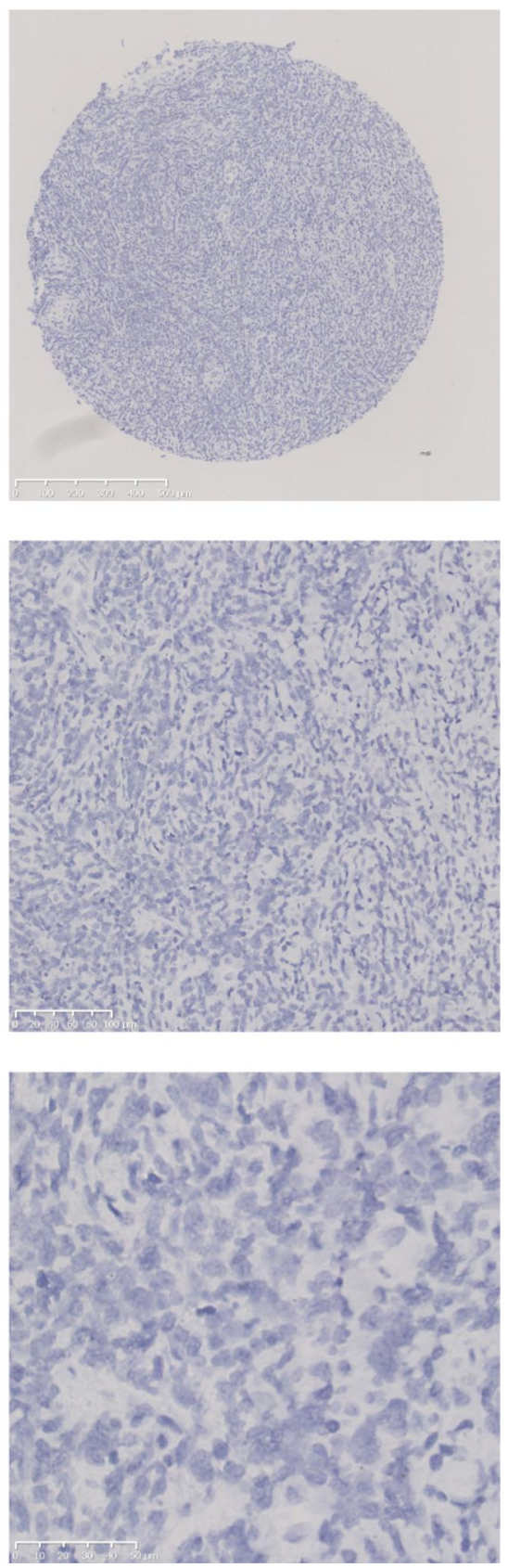

Figure I Representative RNA in situ hybridization images of CD24 mRNA negative expression and positive expression.

\section{Discussion}

Diffuse large B-cell lymphoma is a highly heterogeneous tumor. The heterogeneity causes the differences of biological behavior of tumor and clinical prognosis in DLBCL patients. Currently, rituximab combined with chemotherapy resistance is a tough problem in the treatment of DLBCL, which greatly affects the prognosis of patients.
For patients with refractory DLBCL, the objective response rate was relatively low to the second-line therapy, and the survival was poor. ${ }^{11}$ Therefore, it is of significance to search new potential therapeutic target when resistance of CD20 monoclonal antibody therapy and chemotherapy occurs, so as to improve the treatment effect and prognosis of ABC-DLBCL patients. 
Table I Association Between CD24 Expression Level and Clinicopathological Factors

\begin{tabular}{|c|c|c|c|c|c|}
\hline \multicolumn{2}{|l|}{ Characteristic } & \multirow{3}{*}{$\begin{array}{l}\text { Cases (n) } \\
64 \\
54\end{array}$} & \multirow{3}{*}{$\begin{array}{l}\text { Negative (n) } \\
40 \\
33\end{array}$} & \multirow{3}{*}{$\begin{array}{l}\text { Positive (n) } \\
24 \\
21\end{array}$} & \multirow{3}{*}{$\frac{\mathbf{P} \text { value }}{0.877}$} \\
\hline Gender & Male & & & & \\
\hline & Female & & & & \\
\hline \multirow[t]{2}{*}{ Age } & $\leq 60$ & 81 & 49 & 32 & 0.650 \\
\hline & $>60$ & 37 & 24 & 13 & \\
\hline \multirow[t]{2}{*}{ Stage } & $\mathrm{I}, \mathrm{II}$ & 67 & 43 & 24 & 0.553 \\
\hline & III,IV & 51 & 30 & 21 & \\
\hline \multirow[t]{2}{*}{ ECOG } & $<2$ & 108 & 65 & 43 & $0.314^{*}$ \\
\hline & $\geq 2$ & 10 & 8 & 2 & \\
\hline \multirow[t]{2}{*}{ Extranodal involvement } & $\leq 1$ & 83 & 49 & 34 & 0.330 \\
\hline & $>1$ & 35 & 24 & 11 & \\
\hline \multirow[t]{2}{*}{ LDH } & Normal & 64 & 43 & 21 & 0.195 \\
\hline & Elevated & 54 & 30 & 24 & \\
\hline \multirow[t]{2}{*}{ Treatment response } & $C R$ & 39 & 19 & 20 & 0.039 \\
\hline & Non-CR & 79 & 54 & 25 & \\
\hline
\end{tabular}

Notes: *Fisher's exact test. Bold, $\mathrm{p}$ value is less than 0.05 .

Abbreviations: ECOG, Eastern Cooperative Oncology Group performance status; LDH, lactate dehydrogenase; CR, complete remission.

Table 2 Correlation Between CD24 Expression Level and Clinical Characteristics Obtained from the GEO Database

\begin{tabular}{|c|c|c|c|c|c|}
\hline Characteristic & & Cases (n) & Low Expression (n) & High Expression (n) & p value \\
\hline Gender & $\begin{array}{l}\text { Male } \\
\text { Female }\end{array}$ & $\begin{array}{l}119 \\
80\end{array}$ & $\begin{array}{l}28 \\
22\end{array}$ & $\begin{array}{l}91 \\
58\end{array}$ & 0.527 \\
\hline Age & $\begin{array}{l}\leq 60 \\
>60\end{array}$ & $\begin{array}{l}66 \\
133\end{array}$ & $\begin{array}{l}19 \\
31\end{array}$ & $\begin{array}{l}47 \\
102\end{array}$ & 0.401 \\
\hline Stage & $\begin{array}{l}\mathrm{I}, \mathrm{II} \\
\mathrm{III}, \mathrm{IV}\end{array}$ & $\begin{array}{l}82 \\
117\end{array}$ & $\begin{array}{l}19 \\
31\end{array}$ & $\begin{array}{l}63 \\
86\end{array}$ & 0.595 \\
\hline ECOG & $\begin{array}{l}<2 \\
\geq 2\end{array}$ & $\begin{array}{l}158 \\
41\end{array}$ & $\begin{array}{l}37 \\
13\end{array}$ & $\begin{array}{l}121 \\
28\end{array}$ & 0.276 \\
\hline Extranodal involvement & $\begin{array}{l}\leq 1 \\
>1\end{array}$ & $\begin{array}{l}154 \\
45\end{array}$ & $\begin{array}{l}38 \\
12\end{array}$ & $\begin{array}{l}116 \\
33\end{array}$ & 0.786 \\
\hline LDH & $\begin{array}{l}\text { Normal } \\
\text { Elevated }\end{array}$ & $\begin{array}{l}58 \\
125\end{array}$ & $\begin{array}{l}10 \\
35\end{array}$ & $\begin{array}{l}48 \\
90\end{array}$ & 0.116 \\
\hline Treatment response & $\begin{array}{l}\text { CR } \\
\text { Non-CR }\end{array}$ & $\begin{array}{l}153 \\
46\end{array}$ & $\begin{array}{l}29 \\
21\end{array}$ & $\begin{array}{l}124 \\
25\end{array}$ & 0.0003 \\
\hline
\end{tabular}

Note: Bold, $p$ value is less than 0.05 .

Abbreviations: ECOG, Eastern Cooperative Oncology Group performance status; LDH, lactate dehydrogenase; CR, complete remission.

Recently, CD24 has been found to be connected with tumorigenesis and cancer development, therefore we speculated that CD24 can be a candidate gene for the analysis of treatment response and prognosis evaluation. CD24 gene encodes cluster of differentiation (CD) 24, which is a small glycosyl-phosphatidyl-inositol-anchored molecule. ${ }^{12}$ CD24 is mainly expressed by immune cells, but it is often overexpressed in human tumors. ${ }^{13} \mathrm{CD} 24$ involves in cell proliferation, invasion, metastasis in various cancers and is closely correlated with prognosis of patients. ${ }^{14-17}$ However, the expression level of CD24 in $\mathrm{ABC}-\mathrm{DLBCL}$ is not clear and whether CD24 is involved 


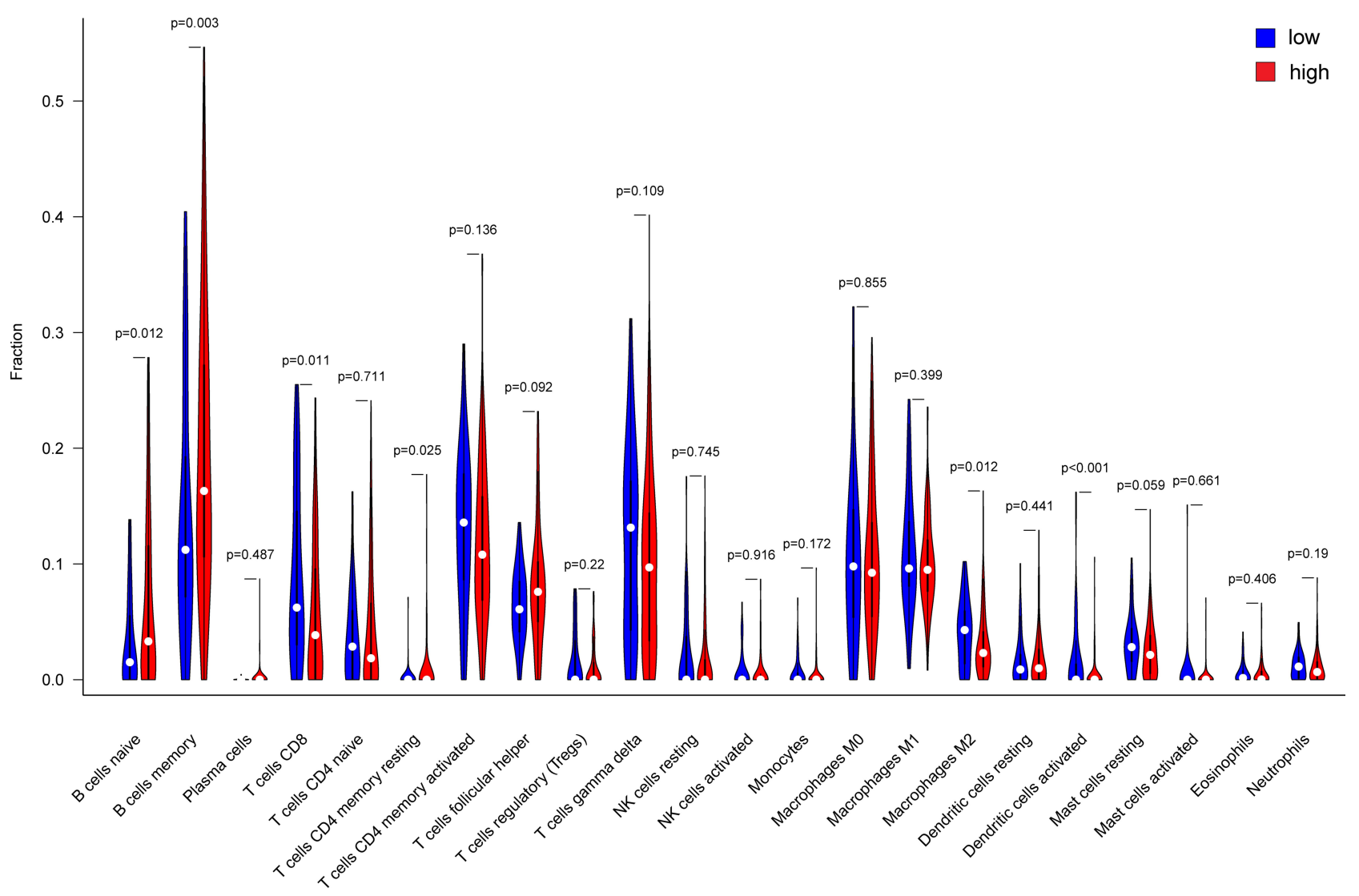

Figure 2 The difference in immune cell infiltration fraction of the 22 distinct leukocyte subsets between CD24 low and high expression group in ABC-DLBCL patients.

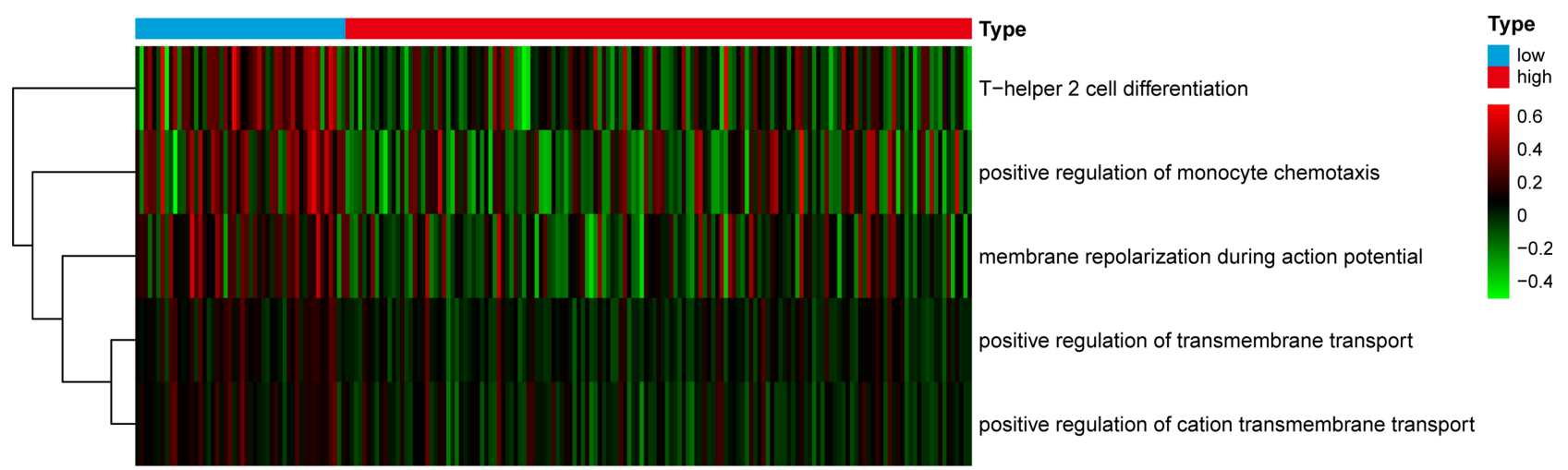

Figure 3 GSVA-derived clustering heatmap of differentially biological process between CD24 low and high expression group in ABC-DLBCL patients.

in the pathogenesis of ABC-DLBCL also remains unknown.

In this study, we initially used RNAscope technology to confirm the expression level of CD24 on tissue microarray of 118 newly diagnosed ABC-DLBCL patients with R-CHOP treatment. RNAscope is a novel RNA in situ hybridization (ISH) technology special for its probe design strategy. The technology achieved simultaneous signal amplification and background suppression, thus realized single-molecule visualization and tissue morphology preservation. ${ }^{18}$ RNAscope technology can detect and evaluate the mRNA expression in the same or adjacent cells with precise quantitative details, and has been widely used in the detection of various pathological tissues. ${ }^{19}$ Thus, it has high specificity and sensitivity to detect CD24 mRNA expression using this method.

The results of RNAscope showed that the positive rate of CD24 mRNA expression was $38.1 \%$. Then, to clarify 
the relationship between CD24 expression level and clinical characteristics of ABC-DLBCL, we compared the clinical characteristics between CD24 negative and positive groups. Notably, the high expression of CD24 was positively correlated with treatment response in $\mathrm{ABC}$ DLBCL patients receiving R-CHOP. Furthermore, in order to expand the sample size, we downloaded the GSE31312 gene set from the GEO database and analyzed the correlation between CD24 expression level and clinical characteristics. We found that the CD24 high expression group had better treatment response and the result was consistent with our research. The present studies have demonstrated the function of CD24 expressed by tumor in promoting immune evasion through its interaction with the inhibitory receptor sialic-acid-binding Ig-like lectin 10 (Siglec-10) expressed by tumor-associated macrophages, and monoclonal antibody blockade of the CD24-Siglec-10 interaction can strongly enhance the phagocytosis of CD24-expressing ovarian cancer and triple-negative breast cancer. $^{20}$ In fact, molecules in cell surface are closely related to tumor immune microenvironment. The cluster of differentiation 47 (CD47), programmed death-ligand 1 (PD-L1), and cytotoxic T lymphocyte antigen 4 (CTLA-4) have been proved to be the innate immune checkpoints that suppress the activity of immune cells so as to help tumor escape from the surveillance of the immune system. ${ }^{21-23}$ In this study, the patients with high expression of CD24 were observed to obtain better curative effect. The phenomenon may be related to the reason that more immune cells respond to the immune effect after the tumor immunosuppressive effect of CD24 was reversed by $\mathrm{R}-\mathrm{CHOP}$ regimen.

Considering CD24 is associated with tumor immune escape, in order to further research the immune mechanism of CD24 in ABC-DLBCL, the CIBERSORT and the LM22 signature matrix were used together to detect the proportions of twenty-two immune cell types between the CD24 high and low expression groups. CIBERSORT is a method for classifying cell composition of complex tissues from their gene expression profiles and it excelled other methods in aspects of noise, unknown mixture content, and closely related cell types. LM22 is a leukocyte gene signature matrix, which is able to differentiate 22 human hematopoietic cell phenotypes. ${ }^{24}$ The distribution of lymphocyte subsets can be displayed effectively by combination of CIBERSORT methodology and LM22 signature matrix. The results revealed that the CD24 high expression patients had significantly lower proportions of
CD8 T cells and nonspecific immune cells, indicating that $\mathrm{T}$ cell immunity and nonspecific immunity of macrophages were suppressed.

Further, in order to make clear the biological process of the immune cells, the GO BP gene sets were analyzed in different groups. Notably, T-helper 2 cell differentiation and monocyte chemotaxis were significantly inhibited in the CD24 high expression group. In terms of immunosuppression mediated by $\mathrm{CD} 24$, sialic acidbinding immunoglobulin-type lectins G/10 (Siglec-G/ 10) is a significant factor enrolled. Siglec-G/10 is the receptor of CD24 and is broadly expressed on B cells, dendritic cells and monocyte subsets. ${ }^{25}$ CD24 interacts with Siglec-10 to negatively regulate the immune response to proteins released by damaged cells. ${ }^{26}$ At present, the study has proved that CD24-Siglec-10 exists in regions of fetal-maternal interactions and CD24 indicated a possible role in mediating immune tolerance at the fetal-maternal boundary. ${ }^{27}$ As a matter of fact, the immune tolerance is similar between pregnancy and cancer.

Current studies have demonstrated that elevated Siglec10 inhibited the protein kinase $\mathrm{C}$ activity induced by phorbol 12-myristate 13-acetate and ionomycin, and repressed the phosphorylation of the tyrosine kinase ZAP-70, thus initially weakened the activation of $\mathrm{T}$ cell in the tumor microenvironment. ${ }^{28}$ Meanwhile, Siglec-G recruited the phosphatase SHP-1, which dephosphorylated the NADPH oxidase component p47 and blocked NOX2 activation. This brought about excessive hydrolysis of exogenous antigens, which caused decreased generation of major histocompatibility complex (MHC) class I-peptide complexes for cross-presentation. ${ }^{29}$ Obviously, CD8 positive dendritic cells (DCs) cross-presented extracellular antigens on MHC class I molecules to start up cytotoxic T lymphocyte (CTL) responses. Therefore, cytotoxic T lymphocyte response was impaired by Siglec-G. For nonspecific immunity, the interaction between Siglec10 and its ligand CD24 suppressed the function of NK cells in hepatocellular carcinoma, shown as decreased granules and cytokine expressions. ${ }^{30}$ Moreover, CD24 monoclonal antibody SWA11 treatment in ovarian carcinoma increased the macrophages infiltration of tumor tissues. $^{31}$ This further proved that antagonizing CD24 expression can improve the immune response of organism to tumors. In summary, the tumor immune response was repressed in the $\mathrm{CD} 24$ high expression group and targeted 
CD24 therapy may be a potential treatment method for patients with R-CHOP resistant ABC-DLBCL.

\section{Conclusion}

CD24 high expression was positively correlated with treatment response in ABC-DLBCL patients receiving R-CHOP. CD24 is a key factor correlated with tumor immune evasion and immune response in ABC-DLBCL. Therefore, CD24 may be a promising signal in treatment and prognosis evaluation in ABC-DLBCL, especially R-CHOP resistant patients. In future, we will design further experiments to verify the function of $\mathrm{CD} 24$, in the hope of improving the prognosis of ABC-DLBCL patients.

\section{Data Sharing Statement}

Data and material will be available upon corresponding author approval. All datasets analyzed for this study are included in the manuscript and the additional files.

\section{Ethics Approval and Consent to Participate}

Informed consent was obtained from all patients and the protocol was approved by the Ethics Committee of Cancer Hospital, Chinese Academy of Medical Sciences.

\section{Author Contributions}

All authors made a significant contribution to the work reported, whether that is in the conception, study design, execution, acquisition of data, analysis and interpretation, or in all these areas; took part in drafting, revising or critically reviewing the article; gave final approval of the version to be published; have agreed on the journal to which the article has been submitted; and agree to be accountable for all aspects of the work.

\section{Funding}

This work was supported by the National Natural Science Foundation of China (81972016) and Beijing Municipal Natural Science Foundation (7162164) \& Chinese Academy of Medical Sciences Innovation Fund for Medical Sciences (2017-I2M-1-013).

\section{Disclosure}

The authors report no conflicts of interest in this work.

\section{References}

1. Roschewski M, Staudt LM, Wilson WH. Diffuse large B-cell lymphoma-treatment approaches in the molecular era. Nat Rev Clin Oncol. 2014;11(1):12-23. doi:10.1038/nrclinonc.2013.197

2. Li S, Young KH, Medeiros LJ. Diffuse large B-cell lymphoma. Pathology. 2018;50(1):74-87. doi:10.1016/j.pathol.2017.09.006

3. Phelan JD, Young RM, Webster DE, et al. A multiprotein supercomplex controlling oncogenic signalling in lymphoma. Nature. 2018;560(7718):387-391. doi:10.1038/s41586-018-0290-0

4. Sehn LH, Gascoyne RD. Diffuse large B-cell lymphoma: optimizing outcome in the context of clinical and biologic heterogeneity. Blood. 2015;125(1):22-32. doi:10.1182/blood-2014-05-577189

5. Van Den Neste E, Schmitz N, Mounier N, et al. Outcome of patients with relapsed diffuse large B-cell lymphoma who fail second-line salvage regimens in the International CORAL study. Bone Marrow Transplant. 2016;51(1):51-57. doi:10.1038/bmt.2015.213

6. Eyvazi S, Kazemi B, Dastmalchi S, et al. Involvement of CD24 in multiple cancer related pathways makes it an interesting new target for cancer therapy. Curr Cancer Drug Targets. 2018;18(4):328-336. doi:10.2174/1570163814666170818125036

7. Hough MR, Rosten PM, Sexton TL, et al. Mapping of CD24 and homologous sequences to multiple chromosomal loci. Genomics. 1994;22(1):154-161. doi:10.1006/geno.1994.1356

8. Lu S, Yao Y, Xu G, et al. CD24 regulates sorafenib resistance via activating autophagy in hepatocellular carcinoma. Cell Death Dis. 2018;9(6):646. doi:10.1038/s41419-018-0681-z

9. Hüser L, Sachindra S, Granados K, et al. SOX2-mediated upregulation of $\mathrm{CD} 24$ promotes adaptive resistance toward targeted therapy in melanoma. Int J Cancer. 2018;143(12):3131-3142. doi:10.1002/ ijc. 31609

10. Ono YJ, Tanabe A, Tanaka T, et al. Met signaling cascade is amplified by the recruitment of phosphorylated met to lipid rafts via CD24 and leads to drug resistance in endometrial cancer cell lines. Mol Cancer Ther. 2015;14(10):2353-2363. doi:10.1158/1535-7163.MCT15-0187

11. Crump M, Neelapu SS, Farooq U, et al. Outcomes in refractory diffuse large B-cell lymphoma: results from the international SCHOLAR-1 study. Blood. 2017;130(16):1800-1808. doi:10.1182/ blood-2017-03-769620

12. Chen GY, Brown NK, Zheng P, et al. Siglec-G/10 in self-nonself discrimination of innate and adaptive immunity. Glycobiology. 2014;24(9):800-806. doi:10.1093/glycob/cwu068

13. Altevogt P, Sammar M, Hüser L, et al. Novel insights into the function of CD24: a driving force in cancer. Int J Cancer. 2021;148 (3):546-559. doi:10.1002/ijc.33249

14. Ooki A, VandenBussche CJ, Kates M, et al. CD24 regulates cancer stem cell (CSC)-like traits and a panel of CSC-related molecules serves as a non-invasive urinary biomarker for the detection of bladder cancer. Br J Cancer. 2018;119(8):961-970. doi:10.1038/ s41416-018-0291-7

15. Soltész B, Lukács J, Szilágyi E, et al. Expression of CD24 in plasma, exosome and ovarian tissue samples of serous ovarian cancer patients. J Biotechnol. 2019;298:16-20. doi:10.1016/j. jbiotec.2019.03.018

16. Wang YC, Wang JL, Kong X, et al. CD24 mediates gastric carcinogenesis and promotes gastric cancer progression via STAT3 activation. Apoptosis. 2014;19(4):643-656. doi:10.1007/s10495-0130949-9

17. Nagare RP, Sneha S, Sidhanth C, et al. Expression of cancer stem cell markers CD24, EPHA1 and CD9 and their correlation with clinical outcome in epithelial ovarian tumours. Cancer Biomark. 2020;28 (3):397-408. doi:10.3233/CBM-201463 
18. Wang F, Flanagan J, Su N, et al. RNAscope: a novel in situ RNA analysis platform for formalin-fixed, paraffin-embedded tissues. J Mol Diagn. 2012;14(1):22-29. doi:10.1016/j.jmoldx.2011.08.002

19. Kersigo J, Pan N, Lederman JD, et al. A RNAscope whole mount approach that can be combined with immunofluorescence to quantify differential distribution of mRNA. Cell Tissue Res. 2018;374 (2):251-262. doi:10.1007/s00441-018-2864-4

20. Barkal AA, Brewer RE, Markovic M, et al. CD24 signalling through macrophage Siglec-10 is a target for cancer immunotherapy. Nature. 2019;572(7769):392-396. doi:10.1038/s41586-019-1456-0

21. Logtenberg MEW, Scheeren FA, Schumacher TN. The CD47-SIRP $\alpha$ immune checkpoint. Immunity. 2020;52(5):742-752. doi:10.1016/j. immuni.2020.04.011

22. Kim JM, Chen DS. Immune escape to PD-L1/PD-1 blockade: seven steps to success (or failure). Ann Oncol. 2016;27(8):1492-1504. doi:10.1093/annonc/mdw217

23. Rowshanravan B, Halliday N, Sansom DM. CTLA-4: a moving target in immunotherapy. Blood. 2018;131(1):58-67. doi:10.1182/ blood-2017-06-741033

24. Newman AM, Liu CL, Green MR, et al. Robust enumeration of cell subsets from tissue expression profiles. Nat Methods. 2015;12 (5):453-457. doi:10.1038/nmeth.3337

25. Yin SS, Gao FH. Molecular mechanism of tumor cell immune escape mediated by CD24/Siglec-10. Front Immunol. 2020;11:1324. doi:10.3389/fimmu.2020.01324
26. Chen GY, Tang J, Zheng P, et al. CD24 and Siglec-10 selectively repress tissue damage-induced immune responses. Science. 2009;323 (5922):1722-1725. doi:10.1126/science.1168988

27. Sammar M, Siwetz M, Meiri H, et al. Expression of CD24 and Siglec-10 in first trimester placenta: implications for immune tolerance at the fetal-maternal interface. Histochem Cell Biol. 2017;147 (5):565-574. doi:10.1007/s00418-016-1531-7

28. Li Y, Zhou J, Zhuo Q, et al. Malignant ascite-derived extracellular vesicles inhibit $\mathrm{T}$ cell activity by upregulating Siglec-10 expression. Cancer Manag Res. 2019;11:7123-7134. doi:10.2147/CMAR. S2 10568

29. Ding Y, Guo Z, Liu Y, et al. The lectin Siglec-G inhibits dendritic cell cross-presentation by impairing MHC class I-peptide complex formation. Nat Immunol. 2016;17(10):1167-1175. doi:10.1038/ ni. 3535

30. Zhang P, Lu X, Tao K, et al. Siglec-10 is associated with survival and natural killer cell dysfunction in hepatocellular carcinoma. J Surg Res. 2015;194(1):107-113. doi:10.1016/j.jss.2014.09.035

31. Salnikov AV, Bretz NP, Perne C, et al. Antibody targeting of CD24 efficiently retards growth and influences cytokine milieu in experimental carcinomas. $B r \quad J$ Cancer. 2013;108(7):1449-1459. doi:10.1038/bjc.2013.102
Pharmacogenomics and Personalized Medicine

\section{Publish your work in this journal}

Pharmacogenomics and Personalized Medicine is an international, peer-reviewed, open access journal characterizing the influence of genotype on pharmacology leading to the development of personalized treatment programs and individualized drug selection for improved safety, efficacy and sustainability. This journal is indexed
Dovepress

on the American Chemical Society's Chemical Abstracts Service (CAS). The manuscript management system is completely online and includes a very quick and fair peer-review system, which is all easy to use. Visit http://www.dovepress.com/testimonials.php to read real quotes from published authors. 